\title{
Gesetze des Denkens? Von Husserls und Freges Psychologismus-Kritik zu einem transzendentalen Kern der Logik
}

David Löwenstein, david.loewenstein@uni-jena.de

In: Zeitschrift für philosophische Forschung

letzte Entwurfsfassung vom 17. September 2019

\begin{abstract}
Deutsch)
Husserl und Frege lehnen den logischen Psychologismus ab, die Auffassung, dass logische Gesetze psychologische ,Gesetze des Denkens' seien. Dieser Aufsatz schlägt eine Lesart dieser berühmten Einwände vor, derzufolge ihre entscheidende Prämisse, die Notwendigkeit der logischen Gesetze, unter Verweis auf eine problematische Metaphysik begründet ist. Diese Prämisse kann jedoch in einer plausibleren Weise gestützt werden, nämlich durch ein transzendentales Argument, das bei der Praxis der rationalen Kritik ansetzt. Dieses Argument wird in einer Auseinandersetzung mit Quines Holismus entwickelt, der die Idee der Notwendigkeit logischer Gesetze zwar zunächst nur noch problematischer zu machen scheint, aber schließlich dennoch für diese Auffassung spricht.
\end{abstract}

\section{Abstract (English)}

Husserl and Frege reject logical psychologism, the view that logical laws are psychological 'laws of thought'. This paper offers an 
account of these famous objections and argues that their crucial premise, the necessity of logical laws, is justified with reference to a problematic metaphysics. However, this premise can be established in a more plausible way, namely via a transcendental argument which starts from the practice of rational criticism. This argument is developed through a discussion of Quine's holism, which at first appears to make the idea of the necessity of logical laws even less plausible, but eventually turns out to speak in favor of this view.

\section{Einleitung}

Logik lässt sich als die Wissenschaft des Begründens, Schließens oder Argumentierens begreifen (vgl. z. B. Strobach 2011, 11): Ein Argument begründet eine These, die Konklusion, mit anderen Thesen, den Prämissen. Die Konklusion soll wahr sein weil die Prämissen wahr sind. Oder anders: Die Prämissen sind wahr, also ist es auch die Konklusion. Dieses ,weil ' bzw. dieses ,also' ist der Gegenstand der Logik.

Logische Gesetze sind daher Gesetze des Schließens - Schlussregeln oder Schlussprinzipien. In diesem Aufsatz geht es mir aber nicht um bestimmte logische Gesetze oder Systeme. Meine Frage lautet: Was sind logische Gesetze überhaupt? Welchen Status haben sie?

Damit haben sich auch Edmund Husserl und Gottlob Frege auseinandergesetzt. So schreibt Husserl im ersten Band seiner in der ersten Auflage bereits 1900 erschienenen Logischen Untersuchungen:

„Wie klingt doch plausibel, was die naheliegende psychologistische Reflexion uns aufdrängen will: Die logischen Gesetze sind Gesetze für Begründungen. Begründungen - was sind sie anderes denn eigenartige Gedankenverläufe der Menschen [...] Wie sollte unter diesen Umständen etwas anderes resultieren als empirische Allgemeinheiten?" (Husserl 1913, 64) 
Husserl stellt hier den Psychologismus vor, nach dem logische Gesetze psychologische Denkgesetze sind. Diese Position greift auch Gottlob Frege im Vorwort seiner Grundgesetze der Arithmetik auf (vgl. Frege 1918, 58):

„,[D]as Wort ,Denkgesetz‘ verleitet zu der Meinung, diese Gesetze regierten in derselben Weise das Denken, wie die Naturgesetze die Vorgänge in der Aussenwelt. Dann können sie nichts anderes als psychologische Gesetze sein; denn das Denken ist ein seelischer Vorgang. Und wenn die Logik mit diesen psychologischen Gesetzen zu thun hätte, so wäre sie ein Theil der Psychologie." (Frege $1893, \mathrm{XV})$

Der Psychologismus ist eine mögliche Antwort auf die Frage nach dem Status der logischen Gesetze als ,Gesetze des Denkens'. Frege und Husserl kritisieren diese Auffassung ganz zurecht. In diesem Aufsatz möchte ich von dieser Kritik ausgehen und dazu drei systematische Thesen verteidigen:

Exegetische These: Freges und Husserls Psychologismus-Kritik hängt wesentlich von der Prämisse ab, dass die logischen Gesetze notwendig wahr sind.

Kritische These: Die Begründung dieser Prämisse durch Frege und Husserl ist problematisch.

Konstruktive These: Die Notwendigkeit der logischen Gesetze lässt sich transzendental begründen.

Ich beginne in Abschnitt 1 mit Freges Psychologismus-Kritik. Dann schlage ich eine detailliertere Betrachtung dieser Überlegungen vor, die auch Husserls Arbeiten einbezieht und die Aspekte der Notwendigkeit der logischen Gesetze (Abschnitt 2), ihrer Idealität (Abschnitt 3) und ihrer Normativität (Abschnitt 4) zunächst unterscheidet und klarer miteinander in Beziehung setzt. ${ }^{1}$ Hier gehe ich durch das Zusammenspiel von Idealität und Normativität schließlich von meiner exegetischen These in den Abschnitten 1 und 2

1 Solche Versuche haben natürlich schon viele unternommen. Auf die umfangreiche historische und systematische Literatur zum Psychologismus, zu Frege und Husserl sowie den Zusammenhängen dieser Positionen kann ich hier jedoch kaum eingehen. Dazu sei hier jedoch zumindest verwiesen auf Føllesdal (1958, 2001), Pivčević (1967), Willard (1972), 
zu meiner kritischen These in den Abschnitten 3 und 4 über. Um meine konstruktive These zu entwickeln, verschärfe ich das so diagnostizierte Problem in Abschnitt 5 noch einmal im Lichte der holistischen Position von Willard Van Orman Quine. Ironischerweise bereitet genau diese Verschärfung aber doch noch einen ungeahnten Weg für eine plausiblere, nämlich transzendentale Begründung der Notwendigkeit logischer Gesetze (Abschnitt 6).

\section{Freges Kritik}

Zunächst zu Freges Psychologismus-Kritik. Frege schreibt:

„Dass die logischen Gesetze Richtschnuren für das Denken sein sollen zur Erreichung der Wahrheit, wird zwar vorweg allgemein zugegeben; aber es geräth nur zu leicht in Vergessenheit. Der Doppelsinn des Wortes ,Gesetz' ist hier verhängnisvoll. In dem einen Sinne besagt es, was ist, in dem andern schreibt es vor, was sein soll. Nur in diesem Sinne können die logischen Gesetze Denkgesetze genannt werden, indem sie festsetzen, wie gedacht werden soll." (Frege 1893, XV)

Wenn der Psychologismus wahr ist, dann sind die logischen Gesetze empirische Gesetze dessen, was Menschen wann denken. Doch trotzdem können sie normative Kraft haben. Frege fährt fort:

„Jedes Gesetz, das besagt, was ist, kann aufgefasst werden als vorschreibend, es solle im Einklange damit gedacht werden, und ist also in dem Sinne ein Denkgesetz. Das gilt von den geometrischen und physikalischen nicht minder als von den logischen." (Frege 1893, XV)

Wenn in dieser Weise alle Gesetze auch normative Auswirkungen haben, dann fragt sich: Was bedeutet dies für die de facto zu beobachtenden psychologischen Gesetzmäßigkeiten? Frege schreibt:

Dummett (1973, 1991a, 1991b), Mohanty (1974, 1977, 1982, 1984, 1997, 2008), McIntyre (1987), Sukale (1988), von Kutschera (1989), Aach (1990), Kusch (1995, 2015), Stepanias (1998), Goldfarb (2001), Parsons (2001), Burge (2005), Macbeth (2005), Hanna (2008), Tieszen (2008), Beyer (2015), Zalta (2017) und Rohr (2019). 
„Man kann dann nur sagen: nach diesen Gesetzen richtet sich im Durchschnitt das Fürwahrhalten der Menschen, jetzt und soweit die Menschen bekannt sind; wenn man also mit dem Durchschnitte im Einklang bleiben will, richte man sich nach ihnen." (Frege 1893, XV)

Doch das bedeutet, dass die logischen Normen des Denkens - sofern es psychologische sind - nicht mehr zwingend sein können. Die logischen Gesetze sind nicht mehr „Richtschnuren [...] zur Erreichung der Wahrheit“, sondern allenfalls zum Erreichen von Übereinstimmung mit dem im Durchschnitt für wahr Gehaltenen. Und das ist natürlich ein wichtiger Unterschied.

Frege betont zurecht: Logische Gesetze sind ,nicht psychologische Gesetze des Fürwahrhaltens, sondern Gesetze des Wahrseins." (Frege 1893, XVI). Es sind notwendige Denkgesetze, da ,sie die allgemeinsten sind, die überall da vorschreiben, wie gedacht werden soll, wo überhaupt gedacht wird." (Frege 1893, XV)

Doch genau das geht verloren, wenn der Psychologismus wahr ist. Natürlich gibt es empirische Gesetze dessen, wie Menschen denken und was sie für wahr halten. Aber diese Gesetze taugen nicht als ,Richtschnuren', als Norman der Erkenntnis. Denn das Ziel ist die Wahrheit - nicht das, was für wahr gehalten wird, von wem und wie vielen auch immer.

Was ist von dieser Kritik zu halten? In komme nun, in den drei folgenden Abschnitten, zu einer etwas detaillierteren Analyse, die auch die Arbeiten von Edmund Husserl einbezieht, und beginne mit der zentralen Prämisse, dass die logischen Gesetze notwendig gelten.

\section{Notwendigkeit}

Obwohl Frege immer wieder auf normative Fragen zu sprechen kommt, lässt sich dies bemerkenswerterweise zunächst einmal ganz einklammern. Wenn logische Gesetze notwendig gelten, dann ergibt sich auch ohne Fragen der Normativität bereits eine direkte Widerlegung des Psychologismus:

Das Argument der Notwendigkeit 
1. Logische Gesetze gelten notwendig.

2. Empirische Gesetze gelten nicht notwendig.

3. Psychologische Gesetze sind empirische Gesetze.

4. Logische Gesetze sind nicht psychologische Gesetze.

Diesen Kern von Freges Überlegungen vertritt auch Husserl. Er schreibt:

„Gewiß gibt die Psychologie nicht mehr [als empirische Allgemeinheiten]. Eben darum kann sie auch nicht jene apodiktisch evidenten und somit überempirischen und absolut exakten Gesetze geben, welche den Kern der Logik ausmachen." (Husserl 1913, 64)

Für dieses Argument ist es natürlich nicht entscheidend, dass die betreffenden empirischen Gesetze psychologische sind und vom Denken handeln. Dasselbe gilt zum Beispiel auch für solche Gesetze der empirischen Sozialwissenschaft oder auch deskriptiven Linguistik, die davon handeln, wer was wann als wahr behauptet. Und das Argument ist natürlich deduktiv gültig. Wer den Psychologismus verteidigen möchte, muss also mindestens eine Prämisse ablehnen.

Und tatsächlich wurde insbesondere die erste Prämisse abgelehnt, also bestritten, dass die logischen Gesetze zwingend gelten: „Hätte sich unser Denken nicht auch ganz anders entwickeln können? Kann es sich nicht weiterhin radikal verändern? Könnten nicht andere Wesen ganz anders denken? Auch wenn das nicht wahrscheinlich scheint: Die Möglichkeit lässt sich nicht ausschließen." Das ist eine relativistische Antwort: Logische Gesetze sind dann nicht notwendig wahr, sondern relativ - abhängig davon, wie de facto gedacht wird. So zumindest verteidigt sich Benno Erdmann in der zweiten, überarbeiteten Auflage seiner Logischen Elementarlehre (vgl. Erdmann 1907, 531 f.) - ein Klassiker des Psychologismus. Erdmann vertritt die noch plausibelste Form eines solchen Relativismus: Logische Gesetze hängen ab von den tatsächlichen Denkregularitäten aller bekannten Menschen. Frege und Husserl diskutieren hier auch Varianten, in denen logische Gesetze sogar von Gruppe zu Gruppe oder im Extremfall von Person zu Person verschieden sind.

Doch alle Spielarten des Relativismus scheitern aus demselben Grund. Wie groß auch immer die Gruppe derer ist, von deren de facto beobachte- 
ten Denkregularitäten die logischen Gesetze abhängen sollen: Es ist immer vorstellbar, dass andere Wesen andere Denkregularitäten aufweisen. In einem solchen Ernstfall macht es der Relativismus unmöglich, sinnvoll zu fragen, welche logischen Gesetze wirklich wahr sind. (vgl. z. B. Husserl 1913, $\S \S 30-40$; Frege 1893, xix; Frege 1918, 69)

Damit wird deutlich wie entscheidend die Prämisse ist, dass die logischen Gesetze notwendig gelten. Doch was berechtigt uns überhaupt zu dieser Annahme? Es zeigt sich: Frege und Husserl begründen sie durch eine Metaphysik idealer, abstrakter Entitäten.

\section{Idealität}

Frege beschreibt die logischen Gesetze als Gesetze des Wahrseins wie folgt:

„Wenn so das Wahrsein unabhängig davon ist, dass es von irgendeinem als wahr anerkannt wird, so sind auch die Gesetze des Wahrseins nicht psychologische Gesetze, sondern Grenzsteine in einem ewigen Grunde befestigt, von unserem Denken überfluthbar zwar, doch nicht verrückbar. Und weil sie das sind, sind sie für unser Denken maassgebend, wenn es die Wahrheit erreichen will." (Frege 1893, XVI)

Dieser „ewige Grund“ ist natürlich nicht in der materiellen Außenwelt zu finden. Doch für Frege handelt es sich ebensowenig um etwas Geistiges. Die Logik handelt von einem dritten Bereich der idealen, abstrakten Gegenstände, zu dem etwa Zahlen gehören und das, was Frege „Gedanken“ nennt, also Pro-

positionen, die Gehalte von Überzeugungen und Behauptungen (vgl. Frege 1918, 69). Und genauso schreibt Husserl:

„Es ist aber absurd, Gesetze, die für Wahrheiten als solche gelten, als Gesetze über Tatsachen zu bezeichnen. Keine Wahrheit ist eine Tatsache, d.i. ein zeitlich Bestimmtes. Eine Wahrheit kann freilich die Bedeutung haben, daß ein Ding ist, ein Zustand besteht, eine Veränderung von statten geht u. dgl. Aber die Wahr- 
heit selbst ist über alle Zeitlichkeit erhaben [...]." (Husserl 1913, $76 \mathrm{f}$.

Husserl verwendet hier zwar das Wort „Tatsache“ anders als Frege, für den eine Tatsache einfach eine wahre Proposition ist (vgl. Frege 1918, 74). Aber in der Sache stimmen beide überein. Propositionen sind absolut und nicht zeitlich bestimmt. Eine Äußerung wie „Jetzt beginnt die Sitzung“ ist zwar zeitlich bestimmt. Doch die damit ausgedrückte Proposition, dass die Sitzung zu dem-und-dem Zeitpunkt beginnt, ist zeitunabhängig, und sie ist zeitunabhängig wahr oder falsch. Logische Gesetze handeln von strukturellen Beziehungen zwischen solchen idealen Propositionen. Deswegen, so führt Husserl aus, sind sie a) absolut exakt (vgl. Husserl 1913, $61 \mathrm{f}$.), sind sie b) a priori, also unabhängig von Erfahrung, evident (vgl. Husserl 1913, 62-64) und c) rein begrifflich wahr, also wahr allein aufgrund der Bedeutung der logischen Begriffe (vgl. Husserl 1913, 73). Und all dies kann nur bedeuten, dass die logischen Gesetze notwendig wahr sind. ${ }^{2}$

\section{Normativität}

Bisher habe ich die Kritik am Psychologismus ganz ohne die normative Rolle der Logik rekonstruiert. Aber passt das zu der oben zitierten Bemerkung Freges: „Nur in diesem Sinne können die logischen Gesetze Denkgesetze genannt werden, indem sie festsetzen, wie gedacht werden soll“? (Frege 1893, XV)

Auch hier vertreten Husserl und Frege dieselbe Position: Die logischen Gesetze selbst sind nicht normativ. Doch sie begründen Normen des Denkens (vgl. Husserl 1913, §§14-16). Freges Bemerkung bedeutet: Wenn man nur dies meint, dann kann man sie Denkgesetze nennen, sie also normativ wenden. Am Beispiel des Modus Ponens: Das Gesetz des Wahrseins „Wenn sowohl ,p' als auch ,wenn p, dann q' wahr ist, ist stets auch ,q' wahr." begründet die Norm der Erkenntnis ,Wer sowohl ,p“ als auch , wenn p, dann q“

2 Dieser Schluss von der Idealität der logischen Gesetze auf ihre notwendige Geltung ist jedoch in Wahrheit gar nicht so klar. Wieso können ideale, abstrakte Gegenstände und ihre Beziehungen nicht ihrerseits kontingent sein? Diese Möglichkeit hat beispielsweise Gideon Rosen (2002) diskutiert. Ich kann das hier jedoch nicht weiter verfolgen. 
für wahr hält, soll ebenfalls ,q für wahr halten."3

Diese Vorrangstellung der logischen Gesetze vor den Erkenntnisnormen bedeutet allerdings auch, dass die Normativität der Logik keine neuen Gründe gegen den Psychologismus liefert. Auch wenn die logischen Gesetze psychologische sind, können sie Erkenntnisnormen begründen. Das Problem besteht erst darin, dass es sich um relative Normen handelt, wo die logischen Erkenntnisnormen doch notwendig gelten. Die Normativität der Logik allein entpuppt sich also im besten Fall als argumentativer Umweg. Wenn die Notwendigkeit logischer Gesetze ohnehin als Prämisse investiert werden muss, dann ist der direkte Weg das Argument der Notwendigkeit.

Ich habe gesagt: ,im besten Fall“ ein Umweg. Denn hier liegt in Wahrheit auch ein ernstes Problem. Und damit komme ich von meiner exegetischen zu meiner kritischen These.

Logische Erkenntnisnormen sollen schließlich nicht nur gelten. Vielmehr muss verständlich werden, dass wir uns an diesen Normen orientieren können. Und dazu reicht es nicht aus, dass wir ihnen im Denken lediglich entsprechen. Vielmehr müssen wir ihnen entsprechen weil wir sie erkennen und uns nach ihnen richten. Und hier rächt sich die Idee der Idealität der logischen Gesetze, denn es entsteht ein Zugänglichkeitsproblem: Lässt sich wirklich verständlich machen, wie wir den weder geistigen noch materiellen dritten Bereich der idealen Gegenstände erkennen können? Frege schreibt, dass wir diese Gegenstände erfassen (vgl. Frege 1918, 76 f.). Doch wie kann dieses Erfassen eine Brücke schlagen zwischen dem Reich des Geistigen in einen davon strikt verschiedenen Bereich des Idealen? Das ist zumindest eine offene Frage. ${ }^{4}$

Aus argumentationstheoretischer Perspektive ergibt sich damit das folgende Bild: Das Argument der Notwendigkeit kritisiert den Psychologismus, erfordert aber die Prämisse der notwendigen Geltung der logischen Gesetze. Der Psychologismus à la Erdmann verwirft diese Prämisse zugunsten eines logischen Relativismus. Frege und Husserl verteidigen sie mit der Konzeption

3 Das genaue Verhältnis dieser Begründungsbeziehung ist in der Zwischenzeit kontrovers diskutiert worden, spätestens seit Gilbert Harmans wegweisendem Buch Change in View (1986). Ein guter Einblick in die jüngste Debatte findet sich bei Steinberger (2016).

$4 \mathrm{Zu}$ den modernen Klassikern die Einwände dieser Art gegenüber Frege formulieren, gehören vor allem die Arbeiten von Michael Dummett (vgl. Fn. 1). 
der Idealgesetze. Doch das macht die Zugänglichkeit und damit die normative Bindung der logischen Gesetze zum Problem. Wie sollte man auf diese Debattenlage reagieren?

\section{Quines Holismus}

Um meine Position zu entwickeln, möchte ich dieses Problem zunächst noch einmal verschärfen, und zwar mit Willard Van Orman Quine. Quine ist nicht nur dafür bekannt, dass er ebenfalls die Idee eines dritten Bereichs idealer Gegenstände ablehnt und stattdessen eine ganz andere, naturalistische Theorie der Bedeutung entwickelt (vgl. z. B. Quine 1960). Mehr noch, Quine schlägt eine ganz grundsätzliche Kritik der Idee vor, dass es überhaupt etwas gibt, das a priori, notwendig und begrifflich-analytisch wahr ist. Die Prämisse, dass die logischen Gesetze notwendig wahr sind, ist dann nicht nur problematisch begründet, sondern sogar widerlegt.

Und warum soll das so sein? Die Antwort findet sich in Quines berühmten Aufsatz „Two Dogmas of Empiricism“ (1951). Dort zeigt Quine, dass sich die Unterscheidung zwischen dem begrifflich Notwendigen und dem empirisch Kontingenten nicht klar ziehen lässt. Stattdessen zeichnet er ein holistisches Bild unserer Erkenntnis. Die beste Ausführung dieser Überlegungen liefert immer noch Quine selbst. Er schreibt:

„The totality of our so-called knowledge or beliefs [...], from the most casual matters of geography and history to the profoundest laws of atomic physics or even of pure mathematics and logic, is a man-made fabric which impinges on experience only along the edges. Or, to change the figure, total science is like a field of force whose boundary conditions are experience. A conflict with experience at the periphery occasions readjustments in the interior of the field. Truth values have to be redistributed over some of our statements. Re-evaluation of some statements entails re-evaluation of others, because of their logical interconnections

- the logical laws being in turn simply certain further statements 
of the system, certain further elements of the field." (Quine 1951, 39)

„If this view is right, [...] it becomes folly to seek a boundary between synthetic statements, which hold contingently on experience, and analytic statements which hold come what may. Any statement can be held true come what may, if we make drastic enough adjustments elsewhere in the system. Even a statement very close to the periphery can be held true in the face of recalcitrant experience by pleading hallucination or by amending certain statements of the kind called logical laws. Conversely, by the same token, no statement is immune to revision." (Quine $1951,40)$

Das bedeutet: Es gibt keine notwendig wahren Sätze, die gelten komme was wolle. Inkonsistenzen im Überzeugungsnetz muss man beseitigen - etwa wenn eine Beobachtung nicht zur akuellen Theorie passt. Doch welche Sätze genau aufgegeben werden sollen, steht nicht fest. Im Prinzip kann es dabei jeden einzelnen Satz treffen, sogar die Gesetze der Logik. In der Praxis ist das natürlich nicht sehr wahrscheinlich. Aber die Möglichkeit besteht.

Und genau das war auch die relativistische Auskunft von Benno Erdmann. Tatsächlich hat Quine selbst für den Psychologismus plädiert, wenn auch nicht immer unter diesem Namen, nämlich im Kontext seiner ,naturalisierten Erkenntnistheorie‘. Tatsächlich trug der Aufsatz „Epistemology Naturalized“ (Quine 1969) zuerst sogar noch den Untertitel „The Case for Psychologism“ (vgl. Kusch 1995, 10 f.). Quine plädiert dafür, Logik und Erkenntnistheorie auf die empirischen Kognitionswissenschaften zurückzuführen, und diese auf die Naturwissenschaften. Überspitzt gesagt: Die naturalisierte Erkenntnistheorie und der historische Psychologismus sitzen in dieser Hinsicht in einem Boot. $^{5}$

Doch Quines Holismus hängt nicht von seinem Naturalismus ab. Und die Notwendigkeit logischer Gesetze steht deswegen auch ganz unabhängig vom

5 Eine ausführlichere und vor allem nuanciertere Diskussion dieser Zusammenhänge und ihrer Konsequenzen findet sich unter anderem bei Kusch $(1995,2015)$, Pelletier et al. (2008), Rott (2008), Wheeler (2008) und van Benthem (2008). 
Naturalismus und vom Psychologismus in Frage. In Wahrheit ist es nämlich unerheblich, wie genau das Überzeugungsnetz geordnet ist, ob die Naturwissenschaft immer das letzte Wort hat, und ob die Inkonsistenzen durch Beobachtung entstehen oder, sagen wir, durch ein intuitives moralisches, ästhetisches oder religiöses Urteil. Entscheidend ist nur, dass bei Konflikten im Prinzip jede Überzeugung aufgegeben werden kann.

Diese Position ist philosophisch umstritten. Doch metaphilosophisch ist sie praktisch unbestreitbar. Es mag gute Gründe für die Unterscheidung zwischen dem notwendig Wahren und dem empirisch Kontingenten geben. Doch diese Gründe sind Teile kontroverser Debatten. Wird in der Philosophie nicht tatsächlich im Prinzip jede beliebige These vertreten oder bestritten - einschließlich aller logischen Gesetze und Systeme?

Diese philosophischen Dissense sind mehr als eine empirische, wissenschaftssoziologische Tatsache. Es sind rationale Dissense. Die Philosophierenden verstehen einander und sie begründen ihre Positionen. Dieses gegenseitige Verstehen kann also nicht die Annahme voraussetzen, dass bestimmte Thesen analytisch und notwendig gelten. Denn genau darüber wird ja diskutiert. ${ }^{6}$ Auch wenn ich das hier nicht weiter verfolgen kann: Der metaphilosophische Blickwinkel macht Quines Holismus tendenziell nur noch stärker.

\section{Der transzendentale Kern der Logik}

Müssen wir die Idee notwendig wahrer logischer Gesetze damit endgültig verabschieden? Ich denke nicht. Um Überzeugungen kritisch zu prüfen und rational zu revidieren, müssen wir auf der Metastufe bereits bestimmte logische Gesetze unterstellen.

Diese konstruktive These lässt sich jedoch nicht nur gegen, sondern ein Stück weit sogar mit Quine begründen. In einem retrospektiven Aufsatz vierzig Jahre nach „Two Dogmas of Empiricism“ scheibt er:

6 In diese Richtung hat etwa auch Timothy Williamson (2007) argumentiert. Innerhalb der Philosophie der Logik wird zudem inzwischen ein Pluralismus verschiedener logischer Systeme diskutiert, der die Notwendigkeit und die normative Verbindlichkeit logischer Gesetze um so mehr gefährdet (vgl. z. B. Steinberger 2019). 
„,[O]ne thing I regret is my needlessly strong statement of holism. [...] In later writings, I have invoked not the whole of science but chunks of it, clusters of sentences just inclusive enough to have critical semantic mass. [...] An observation categorical is a generalization of the form 'Whenever this, that' [...] Now a cluster of sentences has critical semantic mass if it implies an observation categorical; and the experimental check of the cluster of sentences consists in testing the implied categorical by arranging for fulfillment of its observable protasis and seeing whether the apodosis is realized." (Quine 1991, 268)

Quines Rezept lautet also wie folgt: Man nehme eine Menge von Überzeugungen, aus denen eine generelle Subjunktion der Form folgt „Immer wenn in einer Situation $\mathrm{x}$ die Sachverhalte $\mathrm{p}_{1}$ bis $\mathrm{p}_{\mathrm{n}}$ der Fall sind, dann ist in $\mathrm{x}$ auch $\mathrm{q}$ der Fall". Man stelle sicher, dass $\mathrm{p}_{1}$ bis $\mathrm{p}_{\mathrm{n}}$ tatsächlich der Fall sind. Und man prüfe, ob q gilt. Wenn nicht, ist mindestens einer der Ausgangssätze falsch.

Doch erfordert dieses Vorgehen nicht Schlüsse? Und beruhen diese Schlüsse nicht selbst bereits auf auf logischen Schlussprinzipien?

Quine schreibt, dass wir aus der generellen Subjunktion und der Zusatzprämisse, dass die Protasis, also der Wenn-Teil, in einer bestimmten Situation erfüllt ist, darauf schließen sollen, dass die Apodosis, der Dann-Teil, dann ebenfalls erfüllt ist. Wir müssen also erstens aus der generellen Subjunktion die relevante Instanz folgern - eine Allspezialisierung. Und zweitens sollen wir auf die Aussage q schließen - ein Modus Ponens.

Drittens sollen wir uns fragen, ob die erschlossene Aussage q wirklich wahr ist. Und wenn nein? Ein Widerspruch zwischen q und einer anderweitig bestätigten Aussage nicht-q ist nur dann ein Grund zur Revision, wenn der Nichtwiderspruchssatz gilt - das Prinzip „Exakt dieselbe Aussage kann nicht sowohl wahr als auch falsch sein".

Um einzelne oder Mengen von Überzeugungen rational zu kritisieren oder zu revidieren, muss man sich also - selbst nach Quines eigenen Maßstäben - stets auf diese drei fundamentalen logischen Gesetze stützen. Ja, das muss man sogar dann, wenn man ein vermeintliches logisches Gesetz kritisiert, indem man zeigt, dass in einem bestimmten Fall von wahren Prämissen zu einer 
falschen Konklusionen führen kann. Strukturell passiert hier genau dasselbe.

Diese drei Prinzipien gehören zu demjenigen Teil der Logik, der metalogisch unhintergehbar ist. Anders gesagt: Hier liegt der transzendentale Kern der Logik. ,Transzendental' soll hier nur folgendes bedeuten: Die Anwendung und Geltung dieser Gesetze ist eine Bedingung der Möglichkeit des kritischen Denkens überhaupt. Weil kritisches Denken in der Tat möglich ist, sind diese Gesetze notwendig wahr.

Diese Ideen sind natürlich nicht völlig neu. Ähnliches schlägt schon Hans Lenk (1970) im Kontext der inzwischen bereits historischen Debatte um die Begründung der Logik vor. Auch Robert Brandom (1994) leitet die Logik systematisch aus der Praxis des Gebens und Nehmens von Gründen her. Doch mein Argument möchte ich unabhängig von solchen und anderen Vorschlägen eigenständig formulieren und verteigen:

Transzendentalbegründung des Kerns der Logik

1. Vernünftiges kritisches Denken ist tatsächlich möglich.

2. In jedem kritischen Denken muss begründet und widerlegt werden.

3. Wo immer begründet und widerlegt wird, da müssen gewisse logische Kerngesetze unterstellt werden.

4. Transzendentalprinzip: ${ }^{7}$ Was im tatsächlich möglichen vernünftigen Denken unterstellt werden muss, das muss wahr sein, ist also notwendig wahr.

5. Die logischen Kerngesetze sind notwendig wahr.

Dieser transzendentale Kern umfasst die Allspezialisierung, den Modus Ponens und den Nichtwiderspruchssatz - das zeigt Quines Bild -, und sicher auch mehr, etwa den Kettenschluss. Doch werden nicht auch diese Gesetze bestritten? Graham Priest (2008) lehnt zum Beispiel den Nichtwiderspruchssatz ab. Ist mein Vorschlag damit widerlegt?

Nein. Denn bei jedem einzelnen logischen Gesetz bin ich gerne bereit, es im Lichte überzeugender Argumente aufzugeben. Aber erst wenn gezeigt

7 Über die genaue Formulierung und die Plausibilität eines solchen Transzendentalprinzips müsste man natürlich viel ausführlicher diskutieren (vgl. z. B. Tetens 2004, 2006). Ich muss das hier leider einklammern. 
werden kann, dass es für kritisches Denken nicht wesentlich sind. Nun bezweifle ich zwar, dass Priest diesem Anspruch gerecht wird, doch das kann ich hier natürlich nicht mehr begründen. Am Ende ist das aber gar nicht entscheidend. Denn selbst wenn Priest in diesem Punkt am Ende doch Recht behalten sollte, bleibt mein Argument bestehen: Diejenigen Gesetze, die wirklich wesentlich sind für kritisches Denken, sind notwendig wahr. Das, aber auch nur das, zeigt die Transzendentalbegründung. Welche das sind, lohnt es durchaus zu diskutieren. ${ }^{8}$

\section{Schlussbemerkungen}

Damit möchte ich zu meinen drei Kernthesen zurückkehren. Die gerade vorgeschlagene Transzendentalbegründung stärkt Freges und Husserls Kritik am Psychologismus. Denn sie stützt die entscheidende Prämisse, dass logische Gesetze notwendig wahr sind, nämlich mindestens der transzendentale Kern. Auch damit ist der Psychologismus widerlegt. Und zwar ganz ohne Verweis auf einen dritten Bereich idealer Gegenstände.

Zum Abschluss möchte ich kurz ausführen, warum Frege und Husserl diese Transzendentalbegründung durchaus begrüßen könnten.

So erläutert Husserl seine Konzeption einer „reinen“ Logik am Ende des ersten Bandes der Logischen Untersuchungen in klar transzendentalen Begriffen. Nach seiner Auffassung „umspannt die reine Logik in allgemeinster Weise die idealen Bedingungen der Möglichkeit von Wissenschaft überhaupt." (Husserl 1913, 255) Diese transzendentale Wendung lässt sich auch in der weiteren Entwicklung von Husserls Überlegungen aufzeigen, vor allem im zweiten Band der Logischen Untersuchungen (1901), ein Jahr nach der Erstauflage des ersten Bandes, sowie in seinem späteren Werk Formale und Transzendentale Logik (1929). Dort spielt das Subjekt eine größere Rolle für die Logik, jedoch nicht als empirisches, sondern, in Husserls Begriff, als „transzendentales Ego“. Auch wenn diese Dinge historisch und systematisch

\footnotetext{
8 Mit diesem Zugang könnte es sich durchaus lohnen, die Debatten um rivalisierende logische Systeme oder gar einen logischen Pluralismus neu zu rekonstruieren und zu bewerten. Das kann ich hier jedoch natürlich nicht weiter verfolgen.
} 
schwer zu überblicken sind: ${ }^{9}$ Die hier vorgeschlagene Transzendentalbegründung scheint Husserl und der Entwicklung seiner Überlegungen alles andere als fern zu liegen.

$\mathrm{Zu}$ Frege lässt sich festhalten, dass er bereits das logische System der Begriffsschrift aus dem herleitet, was ich hier als transzendentalen Kern der Logik bezeichnet habe. Er verwendet nur den Modus Ponens und führt alle anderen Schlussprinzipien durch Definitionen ein. Frege schreibt: „Man kann nämlich die Wahrheit, die in einer anderen Schlussart liegt, in einem Urtheil aussprechen [...]. So kann ein Schluss in irgendeiner Schlussart auf unsern Fall zurückgeführt werden“" (Frege 1879, § 6, §9), wobei ,unser Fall' hier der Modus Ponens ist. Sobald daneben auch Allspezialisierung und Nichtwiderspruchsprinzip eingeführt wurde, sind auch Kritik und Widerlegung möglich, einschließlich die von Allsätzen, ganz nach Quines Rezept.

Frege betont, mit der Herleitbarkeit logischer Gesetze durch den Modus Ponens solle „keineswegs ein psychologischer Satz ausgesprochen werden“ (Frege 1879, VII). Es gehe hier allein um eine klare und lückenlose Begründung. Dazu bemerkt Frege später:

„,W]arum und mit welchem Recht wir ein logisches Gesetz als wahr anerkennen, kann die Logik nur dadurch beantworten, dass sie es auf andere logische Gesetze zurückführt. Wo das nicht möglich ist, muss sie die Antwort schuldig bleiben. Aus der Logik heraustretend kann man sagen: wir sind durch unsere Natur und die äussern Umstände zum Urtheilen genötigt, und wenn wir urtheilen, können wir dieses Gesetz [...] nicht verwerfen, wir müssen es anerkennen, wenn wir nicht unser Denken in Verwirrung bringen und zuletzt auf jedes Urtheil verzichten wollen." (Frege 1893, XVII)

Hier könnte der Eindruck entstehen, Frege sei inkonsequent: Was soll das anderes sein - einfach nicht anders urteilen zu können, wenn man überhaupt urteilen will -, wenn nicht am Ende doch eine psychologische Unfähigkeit? Doch man kann diese Andeutung durchaus transzendental deuten. Es ist kein

\footnotetext{
9 Siehe hierzu z. B. Gethmann (1989) sowie die eingangs gennante Literatur (vgl. Fn. 1).
} 
Zufall, dass wir ohne den Modus Ponens nicht auskommen. Darauf ist jedes kritische Denken aus transzendentalen Gründen angewiesen.

Aber Frege ist vorsichtiger. Er fährt fort:

„Ich will diese Meinung weder bestreiten noch bestätigen und nur bemerken, dass wir hier keine logische Folgerung haben. Nicht ein Grund des Wahrseins wird angegeben, sondern unseres Fürwahrhaltens." (Frege 1893, XVII)

Doch ein transzendental notwendiges Fürwahrhalten ist nicht irgendein Fürwahrhalten. Natürlich ist das Transzendentalprinzip kein rein logisches Gesetz in Freges Sinne. Es erlaubt jedoch einen Übergang von Fürwahrhalten zu Wahrsein nur in einem ausgezeichneten Fall: Was wir notwendig für wahr halten müssen, damit überhaupt möglich ist, was tatsächlich ist - nämlich unsere Praxis des kritischen Denkens -, das ist auch notwendig wahr.

Die logischen Kerngesetze sind also Gesetze des Denkens. Aber im transzendentalen Sinne des vernünftig nicht Hintergehbaren. Wenn es so etwas gibt wie „Grenzsteine in einem ewigen Grunde befestigt, von unserem Denken überfluthbar zwar, doch nicht verrückbar" (Frege 1893, XVI), dann vielleicht diese. $^{10}$

\section{Literatur}

Aach, John. 1990. Psychologism Reconsidered. A Re-Evaluation of the Arguments of Frege and Husserl. Synthese, 85, 315-338.

van Benthem, Johan. 2008. Logic and Reasoning: Do the facts matter? Studia Logica, 88, 67-84.

Beyer, Christian. 2015. Edmund Husserl. In: Zalta (1995 ff.). Summer 2015 Edition. http://plato.stanford.edu/archives/sum2015/entries/husserl/.

Brandom, Robert. 1994. Making It Explicit. Reasoning, Representing, and Discursive Commitment. Cambridge: Harvard University Press.

\footnotetext{
${ }^{10}$ Für viele konstruktive Gespräche und wohlwollende Verbesserungsvorschläge danke ich Wolfgang Barz, Gregor Betz, Fabian Börchers, Wolfgang Kienzler, Christian Nimtz, Tabea Rohr, Jan Slaby, Holm Tetens und Barbara Vetter.
} 
Burge, Tyler. 2005. Truth, Thought, Reason. Essays on Frege. Oxford: Clarendon Press.

Dummett, Michael. 1973. Frege. Philosophy of Language. New York: Harper \& Row, Publishers.

Dummett, Michael. 1991a. Frege and Other Philosophers. Oxford: Clarendon Press.

Dummett, Michael. 1991b. Frege. Philosophy of Mathematics. London: Duckworth.

Erdmann, Benno. 1907. Logische Elementarlehre. Zweite, überarbeitete Auflage. Halle: Max Niemeyer.

Floyd, Juliet, \& Shieh, Sanford (eds). 2001. Future Pasts. The Analytic Tradition in Twentieth Century Philosophy. Oxford: Oxford University Press.

Føllesdal, Dagfinn. 1958. Husserl und Frege. Ein Beitrag zur Beleuchtung der Entstehung der Phänomenologischen Philosophie. Oslo: I Komisjon Hos H. Aschehoug \& Co. (W. Nygaard).

Føllesdal, Dagfinn. 2001. Bolzano, Frege, and Husserl on Reference and Object. In: Floyd \& Shieh (2001). 67-80.

Frege, Gottlob. 1879. Begriffsschrift, eine der arithmetischen nachgebildete Formelsprache des reinen Denkens. Halle: Verlag von Louis Neubert.

Frege, Gottlob. 1893. Grundgesetze der Arithmetik. Begriffsschriftlich abgeleitet. Jena: Verlag von Hermann Pohle.

Frege, Gottlob. 1918. Der Gedanke. Eine logische Untersuchung. Beiträge zur Philosophie des Deutschen Idealismus, 2, 58-77.

Gethmann, Carl Friedrich. 1989. Phänomenologische Logikfundierung. In: Pöggele, Otto, \& Jamme, Christoph (eds), Phänomenologie im Widerstreit: Zum 50. Todestag Edmund Husserls. Frankfurt am Main: Suhrkamp. 192212.

Goldfarb, Warren. 2001. Frege's Conception of Logic. In: Floyd \& Shieh (2001). 25-41.

Hanna, Robert. 2008. Husserl's Arguments against Logical Psychologism. In: Mayer (2008). 27-42. 
Harman, Gilbert. 1986. Change in View. Principles of Reasoning. Cambridge: MIT Press.

Husserl, Edmund. 1901. Logische Untersuchungen. Zweiter Theil. Untersuchungen zur Phänomenologie und Theorie der Erkenntnis. Halle: Max Niemeyer.

Husserl, Edmund. 1913. Logische Untersuchungen. Erster Band. Prolegomena zur reinen Logik. Zweite, umgearbeitete Auflage. Erstauflage 1900. Halle: Max Niemeyer.

Husserl, Edmund. 1929. Formale und Transzendentale Logik I. Versuch einer Kritik der logischen Vernunft. Halle: Max Niemeyer.

Kusch, Martin. 1995. Psychologism. A Case Study in the the Sociology of Philosophical Knowledge. London \& New York: Routledge.

Kusch, Martin. 2015. Psychologism. In: Zalta (1995 ff.). Winter 2015 Edition. http://plato.stanford.edu/archives/win2015/entries/psychologism/.

von Kutschera, Franz. 1989. Gottlob Frege. Eine Einführung in sein Werk. Berlin \& New York: Walter de Gruyter.

Lenk, Hans. 1970. Philosophische Logikbegründung und rationaler Kritizismus. Zeitschrift für philosophische Forschung, 24, 183-205.

Macbeth, Danielle. 2005. Frege's Logic. Cambridge \& London: Harvard University Press.

Mayer, Verena (ed). 2008. Edmund Husserl. Logische Untersuchungen. Berlin: Akademie Verlag.

McIntyre, Ronald. 1987. Husserl and Frege. The Journal of Philosophy, 84(10), 528-535.

Mohanty, Jitendra Nath. 1974. Frege and Husserl: A New Look at their Relationship. Research in Phenomenology, 4, 51-62.

Mohanty, Jitendra Nath. 1977. Readings on Edmund Husserl's Logical Investigations. Den Haag: Martinus Nijhoff.

Mohanty, Jitendra Nath. 1982. Husserl and Frege. Bloomington: Indiana University Press.

Mohanty, Jitendra Nath. 1984. Husserl, Frege and the Overcoming of Psychologism. In: Cho, Kah Kyung (ed), Philosophy and science in phenomenological perspective. Dordrecht: Martinus Nijhoff Publishers. 143-152. 
Mohanty, Jitendra Nath. 1997. The Concept of 'Psychologism' in Frege and Husserl. Philosophy $\&$ Rhetoric, 30, 271-290.

Mohanty, Jitendra Nath. 2008. The Refutation of Psychologism and Pure Logic. In: The Philosophy of Edmund Husserl: a historical development. New Haven: Yale University Press. 62-83.

Parsons, Charles. 2001. Husserl and the Linguistic Turn. In: Floyd \& Shieh (2001). 123-141.

Pelletier, Francis J., Elio, Renée, \& Hanson, Philip. 2008. Is Logic all in our Heads? From Naturalism to Psychologism. Studia Logica, 88, 3-66.

Pivčević, Edo. 1967. Husserl versus Frege. Mind, 76, 155-165.

Priest, Graham. 2008. An Introduction to Non-Classical Logic. From If to Is. Second Edition. Cambridge: Cambridge University Press.

Quine, Willard Van Orman. 1951. Two Dogmas of Empiricism. The Philosophical Review, 60, 20-43.

Quine, Willard Van Orman. 1960. Word and Object. Cambridge: MIT Press. Quine, Willard Van Orman. 1969. Epistemology Naturalized. In: Ontological Relativity and Other Essays. New York \& London: Columbia University Press. 69-90.

Quine, Willard Van Orman. 1991. Two Dogmas in Retrospect. Canadian Journal of Philosophy, 21, 265-274.

Rohr, Tabea. 2019. Freges Begriff der Logik. Paderborn: Mentis.

Rosen, Gideon. 2002. The Study of Modal Deviance. In: Gendler, Tamar Szabó, \& Hawthorne, John (eds), Conceivability and Possibility. Oxford: Clarendon Press. 283-307.

Rott, Hans. 2008. A New Psychologism in Logic? Reflections from the Point of View of Belief Revision. Studia Logica, 88, 113-136.

Steinberger, Florian. 2016. The Normative Status of Logic. In: Zalta (1995ff.). Spring 2017 Edition. http://plato.stanford.edu/archives/ spr2017/entries/logic-normative/.

Steinberger, Florian. 2019. Logical Pluralism and Logical Normativity. Philosophers' Imprint, 19(12), 1-19.

Stepanias, Markus S. 1998. Frege und Husserl über Urteilen und Denken. Paderborn: Schöningh. 
Strobach, Niko. 2011. Einführung in die Logik. Darmstadt: Wissenschaftliche Buchgesellschaft.

Sukale, Michael. 1988. Logik und Psychologismus. Zeitschrift für allgemeine Wissenschaftstheorie, 19, 62-85.

Tetens, Holm. 2004. Philosophisches Argumentieren. Eine Einführung. München: C.H. Beck.

Tetens, Holm. 2006. Selbstreflexive Physik. Transzendentale Physikbegründung am Beispiel des Strukturenrealismus. Deutsche Zeitschrift für Philosophie, 54, 431-448.

Tieszen, Richard. 2008. Husserl's concept of Pure Logic. In: Mayer (2008). 9-26.

Wheeler, Gregory. 2008. Applied Logic without Psychologism. Studia Logica, 88, 137-156.

Willard, Dallas. 1972. The Paradox of Logical Psychologism: Husserl's Way Out. American Philosophical Quarterly, 9, 94-100.

Williamson, Timothy. 2007. The Philosophy of Philosophy. Malden \& Oxford: Blackwell.

Zalta, Edward N. (ed). 1995 ff.. The Stanford Encyclopedia of Philosophy. Stanford: CSLI.

Zalta, Edward N. 2017. Gottlob Frege. In: Zalta (1995 ff.). Spring 2017 Edition. http://plato.stanford.edu/archives/spr2017/entries/frege/. 